\title{
Geographic and facility-level variation in the use of peritoneal dialysis in Canada: a cohort study
}

\author{
Manish M. Sood MD, Navdeep Tangri MD PhD, Brett Hiebert MSc, Joanne Kappel MD, \\ Allison Dart MD MSc, Adeera Levin MD, Braden Manns MD MSc, Anita Molzahn RN PhD, \\ David Naimark MD MSc, Sharon J. Nessim MD MSc, Claudio Rigatto MD MSc, \\ Steven D. Soroka MD, Michael Zappitelli MD MSc, Paul Komenda MD MHA; on behalf \\ of The Canadian Kidney Knowledge Translation and Generation Network
}

\section{Abstract}

Background: Peritoneal dialysis is associated with similar survival and similar improvement in quality of life and is less costly compared with in-centre hemodialysis. We examined facility and geographic variation in the use of peritoneal dialysis in Canada.

Methods: We analyzed data from the Canadian Organ Replacement Register for the period January 2001 to December 2010 . We identified patients for whom peritoneal dialysis was the primary modality at 90 days after initiation of dialysis. We used multilevel models to evaluate variation in use of peritoneal dialysis by facility and geographic region.

Results: We analyzed data for 31778 incident dialysis patients at 56 facilities in 13 geographic regions across Canada. Use of peritoneal dialysis at 90 days varied considerably across geographic regions (range $19.8 \%-36.1 \%$ ) and declined over time, from $28.8 \%$ in 2001 to $22.5 \%$ in 2010. After adjustment for case mix and facility-level quality indicators, $9.3 \%$ and $3.4 \%$ of the variability was attributable to facility and geographic factors, respectively. In adjusted models, there was a substantial difference between geographic regions with the lowest and highest peritoneal dialysis use (odds ratio for high use 1.51, 95\% confidence interval [Cl] $1.33-1.73 \mathrm{v}$. odds ratio for low use $0.69,95 \% \mathrm{Cl} 0.60-0.79)$.

Interpretation: In Canada, substantial variability in the use of peritoneal dialysis attributable to facility and geographic region was not explained by differences in patient case mix. An opportunity exists to optimize use of this cost-effective therapy through changes in policy and standardization of criteria for initiation of peritoneal dialysis.

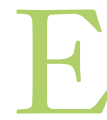

very year, about 3000 patients will start some form of long-term dialysis therapy in Canada, a number that is increasing steadily at $0.5 \%$ to $1.0 \%$ per year. $^{1}$ In-centre (often hospital-based) intermittent hemodialysis is the most common form of dialysis in Canada, accounting for roughly $70 \%$ of all patients treated. However, home dialysis modalities such as peritoneal dialysis are available in most Canadian centres. Mortality rates associated with peritoneal dialysis are comparable to those associated with incentre hemodialysis, and peritoneal dialysis may offer several other advantages, including improvements in quality of life, higher patient satisfaction, maintenance of independence, ability to travel and improved hemodynamic stability. ${ }^{2-10}$ The various dialysis modalities are often complementary, and patients may transition among modalities over their lifetimes. From the perspective of health care payers, there are substantial cost advantages to peritoneal dialysis, with average annual savings of about $\$ 20000$ per patient, relative to in-centre hemodialysis. Given similar patient sur- vival and cost-effectiveness and other advantages, many Canadian jurisdictions have informally adopted a "peritoneal dialysis first" approach for the delivery of renal replacement therapy.

Numerous factors at the patient, facility and geographic levels have been reported to independently influence the use of peritoneal dialysis. A patient's suitability for peritoneal

Competing interests: Manish Sood has served on advisory boards for Roche and Amgen and has received honoraria from Roche, Amgen and Sanofi. Sharon Nessim has served on advisory boards for Baxter Healthcare Canada and has received speaking honoraria from Baxter Healthcare Canada and Merck Frost. Paul Komenda sits on the Takeda advisory board. No other competing interests declared.

This article has been peer reviewed.

Correspondence to: Manish Sood, msood99@gmail.com

CMAJ Open 2014.DOI:10.9778/cmajo.20130050 
dialysis may be determined by the presence of comorbidities, such as cardiac disease and obesity, and by demographic factors, such as ethnic origin, rural residence and age. ${ }^{11-16}$ Regional and facility-level factors such as local expertise, individual physicians' opinions and knowledge of peritoneal dialysis, size of the dialysis program, presence and duration of predialysis care, reimbursement and standardized education about dialysis modalities are associated with use of peritoneal dialysis. ${ }^{17-21}$ Considering the multitude of factors that may influence use of this type of renal replacement therapy, along with the lack of standardized, evidence-based criteria for determining a patient's suitability, it follows that there may be considerable variation in use of peritoneal dialysis across facilities and geographic regions, and the optimal application of peritoneal dialysis remains unknown. ${ }^{17}$ Understanding to what extent patient-level versus geographic and facility-level variables account for the variation in use of peritoneal dialysis would aid in the design of future policy interventions. For example, variation due to geographic or facility-level differences might be successfully addressed by health policy, whereas variation in peritoneal dialysis uptake attributable to patients' biological characteristics would likely not. To date, no studies have quantified the relative contributions of facility and geographic factors to the decision to use peritoneal dialysis, but this information would facilitate the development of quality improvement programs.

Our objective was to determine the degree of variation in use of peritoneal dialysis across geographic regions in Canada and to estimate the influence of facility-level and regional characteristics on any such variation. We hypothesized that significant variability occurs at the facility and regional level, which would be potentially amenable to policy interventions.

\section{Methods}

\section{Population and data sources}

We obtained data for incident dialysis patients (patients for whom dialysis had been newly initiated) who were registered in the Canadian Organ Replacement Register from January 2001 to December 2010. We included all adult patients (> $18 \mathrm{yr}$ ) whose modality of dialysis at the time of initiation was known. Data were collected until patients died, received a kidney transplant or were lost to follow-up or until the end of study period (Dec. 31, 2010). The Canadian Organ Replacement Register is a validated registry that records data for all patients with end-stage renal disease in Canada (demographic characteristics, comorbidities, dialysis modality, vascular access, transplantation and death). ${ }^{1,22}$ Data from Quebec were not included because of the need for additional ethics and data permissions.

\section{Cohort definitions}

The cohort definitions have been previously reported. ${ }^{11}$ In brief, we identified patients for whom peritoneal dialysis was listed as the primary modality at 90 days after initiation of dialysis. The period of 90 days was chosen to allow time for medical stabilization and receipt of education about dialysis modalities for patients with short periods of predialysis care, such as those with acute kidney injury leading to end-stage renal disease. Patients' demographic characteristics (age, sex, ethnic origin, body mass index) and comorbidities (angina, acute coronary syndrome, pulmonary edema, diabetes mellitus, stroke, peripheral vascular disease, malignancy, lung disease, hypertension medications, current smoker, coronary artery bypass graft, serious illness) were captured at the time of dialysis initiation. Serious illness was defined as any illness that could shorten life expectancy to less than 5 years. We used the interval between the date of a patient's first visit with a nephrologist and the date of dialysis initiation to estimate the length of predialysis nephrology care. We calculated distance to the dialysis centre as the direct linear distance (in kilometres) from the patient's primary residence (by postal code) at the time of dialysis initiation to the nearest dialysis provider using Vincenty's formula, ${ }^{23}$ with stratification according to a previous Canadian report. ${ }^{24}$ Individual patients and dialysis facilities were de-identified. Geographic regions were created by the Canadian Organ Replacement Register and were designed to maintain anonymity of participating centres. The geographic regions were Atlantic (consisting of 2 regions: Newfoundland and Labrador, New Brunswick and Prince Edward Island combined, and Nova Scotia), Ontario (subdivided into greater Toronto and northern, eastern and western regions), Manitoba, Saskatchewan, Alberta (subdivided into northern and south regions) and British Columbia (subdivided into Vancouver, eastern region and "other"). We also extracted certain laboratory values (hemoglobin, albumin and phosphate) that were recorded at the time of dialysis initiation.

Facility-level variables were based on measures associated with clinical outcomes. ${ }^{25-28}$ These variables included the proportion of patients who received dialysis via a central venous catheter, whether the facility offered renal transplantation, mean hemoglobin and phosphate levels at dialysis initiation for patients treated at the centre, mean distance (in kilometres) between patients' primary residence and nearest dialysis facility, and the size of the centre (i.e., total number of patients treated over the study period).

In separate sensitivity analyses, we identified patients who initiated dialysis with the peritoneal modality (i.e., peritoneal dialysis from day 1 of dialysis therapy) and patients who started peritoneal dialysis at any time during the study period (i.e., not limited to initiation in the first 90 days).

\section{Statistical analysis}

We compared patient, facility and geographic characteristics across patients receiving peritoneal dialysis and those receiving intermittent hemodialysis. Continuous variables of interest were summarized as means (with standard deviations) or medians (with interquartile ranges), as appropriate. Differences in characteristics were determined by the Student $t$ test or the Mann-Whitney $U$ test for continuous variables and the $\chi^{2}$ test for dichotomous variables.

We examined facility and geographic variations using multilevel modelling, with patients nested within facilities and 
facilities nested within geographic regions. We used a 3-level logistic model to assess variables associated with peritoneal dialysis. Variation in the multilevel model was determined by intraclass correlation. ${ }^{29,30}$ The intraclass correlation coefficient is the ratio of variance between levels in a multilevel model to variance within each level. In our study, the intraclass correlation coefficient determined the proportion of variation in use of peritoneal dialysis that was due to being a member of a particular group, such as patient group, facility or geographic region, and is reported as a percentage. ${ }^{31,32}$ Only facilities that offered peritoneal dialysis were included in the analysis. Models were adjusted for facility-level factors (percentage of patients with central venous catheter, transplantation facility, mean hemoglobin level, mean phosphate level, number of patients) and patient case mix (age, sex, body mass index, ethnic origin, comorbidities, distance to facility, duration of predialysis care, hemoglobin, albumin and phosphate). We then created unadjusted, fully adjusted and reduced models. Variables were included in the reduced models if the $p$ value was less than 0.01 in the full model and were retained if the $p$ value remained less than 0.05 in the reduced model. Finally, we calculated the odds ratios for initiation of early dialysis by geographic region in a separate 2-level multilevel logistic model adjusted for patient case mix and facility-level factors listed previously. We created separate multilevel models to examine use of peritoneal dialysis at dialysis initiation and use of peritoneal dialysis at any time.

We employed multiple imputation for missing values, with a random draw from the predictive distribution from an imputation model repeated 10 times. ${ }^{33}$ Analyses were performed using PASW version 18 (IBM, Armonk, New York) and SAS version 9.3 (SAS Institute Inc., Cary, North Carolina).
All hypothesis tests were 2-sided with statistical significance determined by a $p$ value of less than 0.05 .

\section{Results}

Over the 10-year study period, a total of 31778 incident dialysis patients in 56 facilities across 13 geographic regions met our inclusion criteria. Patients who were receiving peritoneal dialysis by 90 days were younger, more likely to be female and more likely to have received predialysis care and had fewer comorbid conditions (Table 1). Patients receiving peritoneal dialysis were also more likely to have higher hemoglobin and lower serum phosphate and to live further from a dialysis facility. Aboriginal patients accounted for a smaller proportion of patients receiving peritoneal dialysis (compared with those not receiving peritoneal dialysis), whereas East Asian patients accounted for a higher proportion of patients receiving peritoneal dialysis (compared with those not receiving peritoneal dialysis).

Of the patients included in the analysis, 8091 (25.5\%) were receiving peritoneal dialysis at 90 days. There was a downward trend in use of peritoneal dialysis, from $28.8 \%$ $(767 / 2663)$ in 2001 to $22.5 \%(877 / 3898)$ in 2010 . Use of peritoneal dialysis was highest in the greater Toronto region (36.1\%), followed by Vancouver (32.6\%) and eastern British Columbia (28.5\%) (Figure 1). The lowest use of peritoneal dialysis was observed in northern Alberta (20.9\%), the Atlantic provinces excluding Nova Scotia (20.3\%) and eastern Ontario (19.8\%).

In an unadjusted (intercept-only) model, dialysis facility and geographic region accounted for $13.8 \%$ and $1.1 \%$ of the variation in use of peritoneal dialysis (Table 2). After

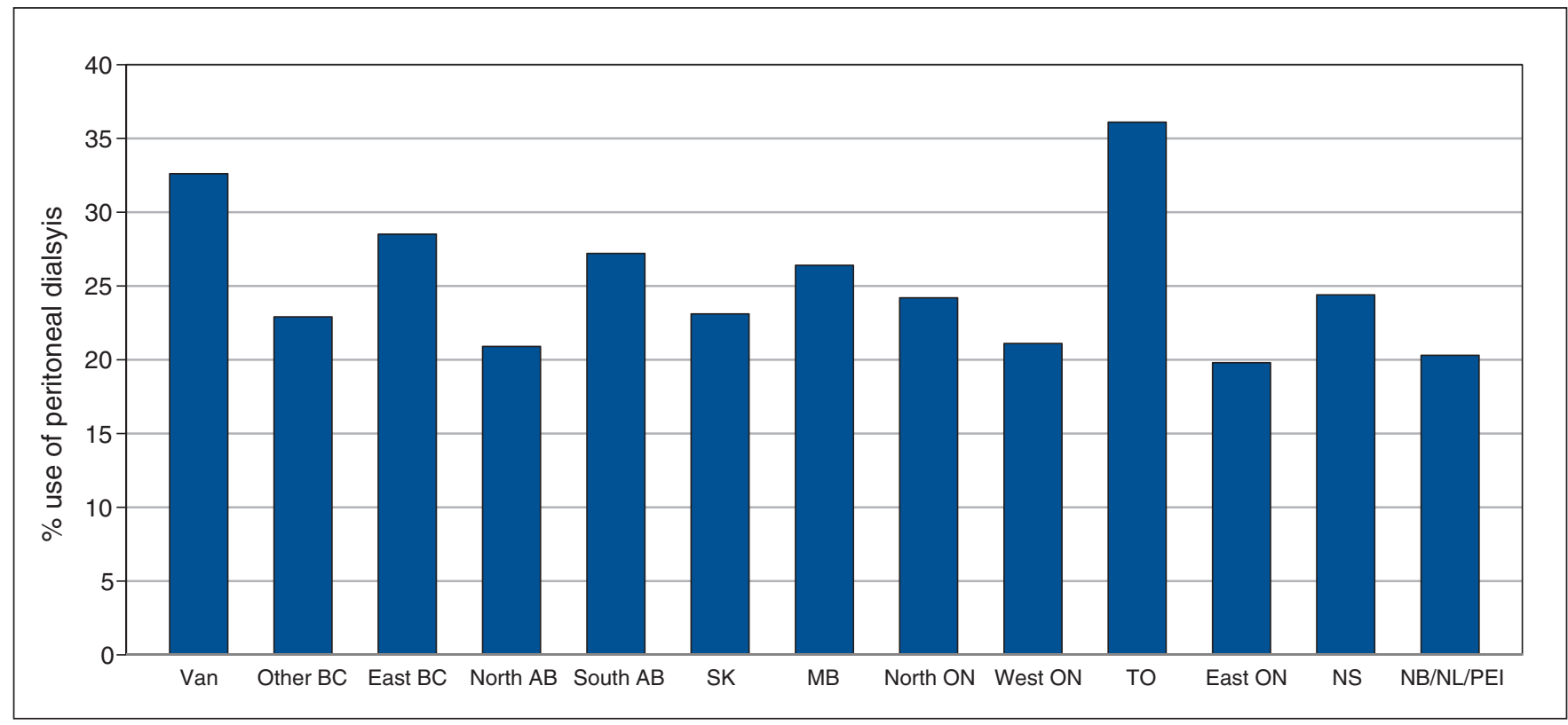

Figure 1: Crude proportion of patients undergoing peritoneal dialysis by 90 days in each geographic region (from west to east). For each region, the denominator was all patients who received renal replacement therapy (hemodialysis, peritoneal dialysis or pre-emptive transplantation) dur-

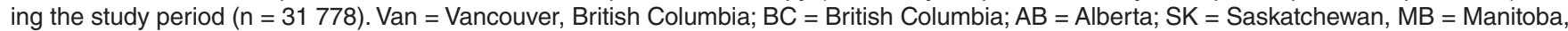
$\mathrm{ON}=$ Ontario; $\mathrm{TO}=$ Toronto, Ontario; NS = Nova Scotia; NB = New Brunswick; NL = Newfoundland and Labrador; PEI = Prince Edward Island. 
Table 1: Baseline characteristics of patients with and without peritoneal dialysis by 90 days after dialysis initiation

\begin{tabular}{|c|c|c|c|}
\hline Characteristic & $\begin{array}{l}\text { Peritoneal dialysis } \\
\qquad n=8091\end{array}$ & $\begin{array}{c}\text { No peritoneal dialysis } \\
\qquad n=23687\end{array}$ & $p$ value $^{*}$ \\
\hline Age, yr, mean \pm SD & $60.3 \pm 16.4$ & $65.3 \pm 15.3$ & $<0.001$ \\
\hline Sex, no. (\%) female & 3431 (42.4) & $9498(40.1)$ & $<0.001$ \\
\hline Body mass index, mean \pm SD & $26.8 \pm 5.7$ & $27.7 \pm 6.8$ & $<0.001$ \\
\hline Self-reported ethnic origin, no. (\%) of patients & & & $<0.001$ \\
\hline White & $5542(68.5)$ & 17268 (72.9) & \\
\hline East Asian & $712(8.8)$ & 1374 & \\
\hline Black & $291 \quad(3.6)$ & $734 \quad(3.1)$ & \\
\hline South Asian & $364 \quad(4.5)$ & $900 \quad(3.8)$ & \\
\hline Aboriginal & $429 \quad(5.3)$ & $1327 \quad(5.6)$ & \\
\hline Other & $396 \quad(4.9)$ & $1350 \quad(5.7)$ & \\
\hline Unknown & $357 \quad(4.4)$ & $734 \quad(3.1)$ & \\
\hline Predialysis care $>30 \mathrm{~d}$, no. (\%) of patients & $7387(91.3)$ & $17481(73.8)$ & $<0.001$ \\
\hline \multicolumn{4}{|l|}{ Comorbidities, no. (\%) of patients } \\
\hline Angina & $1416(17.5)$ & $6088(25.7)$ & $<0.001$ \\
\hline Acute coronary syndrome & $1448(17.9)$ & $6040(25.5)$ & $<0.001$ \\
\hline Pulmonary edema & $1384(17.1)$ & $7201(30.4)$ & $<0.001$ \\
\hline Diabetes mellitus & $3495(43.2)$ & $11299(47.7)$ & $<0.001$ \\
\hline Stroke & $930(11.5)$ & $3908(16.5)$ & $<0.001$ \\
\hline Peripheral vascular disease & $1246(15.4)$ & $5353(22.6)$ & $<0.001$ \\
\hline Malignancy & $752 \quad(9.3)$ & $3600(15.2)$ & $<0.001$ \\
\hline Lung disease & $615 \quad(7.6)$ & $3340(14.1)$ & $<0.001$ \\
\hline Hypertension medications & 7217 (89.2) & $20181(85.2)$ & $<0.001$ \\
\hline Current smoker & $955(11.8)$ & $3174(13.4)$ & $<0.001$ \\
\hline Coronary artery bypass graft & $971(12.0)$ & $3908(16.5)$ & $<0.001$ \\
\hline Serious illness & $761 \quad(9.4)$ & $3743(15.8)$ & $<0.001$ \\
\hline No. of comorbidities, mean $\pm \mathrm{SD}$ & $2.6 \pm 1.7$ & $3.3 \pm 2.0$ & $<0.001$ \\
\hline Cause of ESRD, no. (\%) of patients & & & $<0.001$ \\
\hline Hypertension & $1398(17.3)$ & $4815(20.3)$ & \\
\hline Diabetes mellitus & $2904(35.9)$ & $8533(36.0)$ & \\
\hline Glomerulonephritis & $1578(19.5)$ & $3486(14.7)$ & \\
\hline Obstruction & $178 \quad(2.2)$ & $640 \quad(2.7)$ & \\
\hline Interstitial & $185 \quad(2.3)$ & $616 \quad(2.6)$ & \\
\hline Polycystic kidney disease & $499 \quad(6.2)$ & $876 \quad(3.7)$ & \\
\hline Other & $801 \quad(9.9)$ & $2561(10.8)$ & \\
\hline Unknown & $548 \quad(6.8)$ & $2160 \quad(9.1)$ & \\
\hline Hemoglobin, g/L, mean \pm SD & $108.1 \pm 16.5$ & $100.4 \pm 17.4$ & $<0.001$ \\
\hline Phosphate, $\mathrm{mmol} / \mathrm{L}$, mean $\pm \mathrm{SD}$ & $1.82 \pm 0.56$ & $1.94 \pm 0.69$ & $<0.001$ \\
\hline Distance to facility, $\mathrm{km}$, median (IQR) & $15.6(6.3-66.4)$ & $10.6(4.6-44.2)$ & $<0.001$ \\
\hline$<50$ & $5696(70.4)$ & $18192(76.8)$ & $<0.001$ \\
\hline $50-150$ & $1440(17.8)$ & $3600(15.2)$ & \\
\hline$>150$ & $955(11.8)$ & $1895 \quad(8.0)$ & \\
\hline Geographic region, $\nmid$ no. (\%) of patients & & & $<0.001$ \\
\hline Atlantic & $752 \quad(9.3)$ & $2392(10.1)$ & \\
\hline Ontario & $4313(53.3)$ & $12791(54.0)$ & \\
\hline Prairies & $1278(15.8)$ & $4832(20.4)$ & \\
\hline British Columbia & $1748(21.6)$ & $3672(15.5)$ & \\
\hline \multicolumn{4}{|c|}{$\begin{array}{l}\text { Note: ESRD }=\text { end-stage renal disease, IQR }=\text { interquartile range, } \mathrm{SD}=\text { standard deviation. } \\
\text { * } \mathrm{Statistical} \text { testing for all continuous variables was by Student } t \text { test, except for median distance to facility, for which the Mann- } \\
\text { Whitney } U \text { test was used. Categorical variables were examined by the } \chi^{2} \text { test. } \\
\text { †Atlantic provinces consist of Prince Edward Island, Nova Scotia, New Brunswick, and Newfoundland and Labrador. Prairie provinces } \\
\text { consist of Manitoba, Saskatchewan, and Alberta. For the purposes of data analysis, Ontario was subdivided into } 4 \text { regions, British } \\
\text { Columbia was subdivided into } 3 \text { regions, Alberta was subdivided into } 2 \text { regions, and data for Prince Edward Island, New Brunswick, } \\
\text { and Newfoundland and Labrador were combined. }\end{array}$} \\
\hline
\end{tabular}




\section{OPEN}

adjustment for case mix and facility-level factors, the variation was $9.3 \%$ and $3.4 \%$, respectively. Results were similar in a reduced model that included only statistically significant variables. Additional models examining peritoneal dialysis from initiation of therapy and use of peritoneal dialysis at any time demonstrated comparable levels of facility and geographic variation (Table 2). Notably, facility and geographic variation declined with length of the assessment period of peritoneal dialysis use (peritoneal dialysis at any time $<$ peritoneal dialysis at 90 days $<$ peritoneal dialysis on initiation of therapy).

Table 3 shows factors associated with use of peritoneal dialysis at 90 days. Facilities with greater use of central venous catheters had lower use of peritoneal dialysis. Patient characteristics associated with use of peritoneal dialysis at 90 days included younger age, female sex, Asian ethnic origin, lower body mass index, less comorbidity, greater distance from a dialysis facility and predialysis care. Higher hemoglobin level and lower phosphate level were also associated with use of peritoneal dialysis.

Figure 2 shows the odds ratios for use of peritoneal dialysis adjusted for facility and case mix in different geographic regions. With Manitoba as the referent, there was more than

\section{Table 2: Multilevel model of unadjusted and adjusted variation in use of peritoneal dialysis, at the facility and geographic levels*}

\begin{tabular}{|c|c|c|}
\hline \multirow[b]{2}{*}{ Model } & \multicolumn{2}{|c|}{$\begin{array}{l}\text { Level of variation; } \\
\text { intraclass correlation coefficient, \% }\end{array}$} \\
\hline & $\begin{array}{l}\text { Geographic } \\
\text { region }\end{array}$ & Facility \\
\hline \multicolumn{3}{|c|}{ Peritoneal dialysis at $90 \mathrm{~d}$} \\
\hline Unadjusted & 1.1 & 13.8 \\
\hline Fully adjusted & 3.4 & 9.3 \\
\hline Reduced & 3.2 & 8.8 \\
\hline \multicolumn{3}{|c|}{$\begin{array}{l}\text { Peritoneal dialysis } \\
\text { at initiation of therapy }\end{array}$} \\
\hline Unadjusted & 1.1 & 15.7 \\
\hline Fully adjusted & 4.0 & 11.4 \\
\hline Reduced & 3.7 & 10.7 \\
\hline \multicolumn{3}{|c|}{$\begin{array}{l}\text { Peritoneal dialysis } \\
\text { at any time }\end{array}$} \\
\hline Unadjusted & 0.6 & 11.8 \\
\hline Fully adjusted & 2.0 & 7.7 \\
\hline Reduced & 1.7 & 7.3 \\
\hline \multicolumn{3}{|c|}{$\begin{array}{l}\text { *The analysis involved } 13 \text { geographic regions, } 56 \text { facilities and } 31778 \text { patients. } \\
\text { The fully adjusted model included the following covariates: for facility-level } \\
\text { factors, percentage of patients with a central venous catheter, transplantation } \\
\text { facility, mean serum hemoglobin and phosphate, mean distance of patient's } \\
\text { residence from nearest dialysis centre and number of patients served; for patient } \\
\text { case mix, age, sex, body mass index, ethnic origin, comorbidities, distance to } \\
\text { facility, length of predialysis care, serum hemoglobin, serum phosphate and } \\
\text { serum albumin. The reduced model included the following covariates: for facility- } \\
\text { level factors, percentage of patients with a central venous catheter; and for } \\
\text { patient case mix, age, sex, body mass index, ethnic origin, cause of end-stage } \\
\text { renal disease, comorbidities, distance to facility, length of predialysis care, serum } \\
\text { hemoglobin and serum phosphate. }\end{array}$} \\
\hline
\end{tabular}

2 -fold difference in use of peritoneal dialysis between high-use regions (Toronto and Vancouver) and low-use regions (northern Alberta, western and eastern Ontario, other areas of British Columbia and the Atlantic provinces excluding Nova Scotia).

Table 3: Variables associated with use of peritoneal dialysis (reduced multilevel model)

Variable OR $(95 \% \mathrm{Cl})$

Facility-level

Use of central venous catheter,

$0.96(0.93-0.99)$ per $1 \%$ increase

\section{Patient-level}

Age, per yr

$0.98(0.98-0.98)$

Sex, male

$0.93(0.88-0.98)$

Body mass index, per unit increase

$0.98(0.97-0.98)$

Hemoglobin, per 1-g/L increase

$1.02(1.02-1.02)$

Phosphate, per 0.5-mmol/L increase

$0.91(0.88-0.93)$

Predialysis care $>30 \mathrm{~d}$

$3.04(2.78-3.31)$

Comorbidities

\begin{tabular}{ll} 
Angina & $0.86(0.80-0.93)$ \\
\hline Pulmonary edema & $0.71(0.66-0.77)$ \\
\hline Diabetes mellitus & $0.74(0.67-0.82)$ \\
Stroke & $0.81(0.74-0.88)$ \\
\hline Peripheral vascular disease & $0.86(0.79-0.92)$ \\
\hline Malignancy & $0.74(0.68-0.82)$ \\
Lung disease & $0.71(0.64-0.78)$ \\
Hypertension medications & $1.38(1.26-1.50)$ \\
Serious illness & $0.66(0.60-0.72)$
\end{tabular}

Distance from facility, $\mathrm{km}$

$<50$ (ref) $\quad 1.00$

50-150

$1.83(1.68-1.98)$

$>150$

$2.52(2.26-2.80)$

Cause of end-stage renal disease

Hypertension

$1.02(0.90-1.15)$

Diabetes mellitus

$1.48(1.28-1.70)$

Glomerulonephritis

$1.29(1.14-1.47)$

Obstruction

$0.86(0.70-1.06)$

Interstitial

$0.85(0.69-1.03)$

Polycystic kidney disease

$1.03(0.87-1.20)$

Other

$1.02(0.89-1.17)$

Ethnic origin

White (ref) 1.00

East Asian $1.18(1.05-1.32)$

Black $1.01(0.86-1.18)$

South Asian $0.90(0.78-1.04)$

Aboriginal $\quad 0.70(0.61-0.80)$

Other

$1.12(0.96-1.29)$

Unknown

$0.96(0.84-1.09)$

Note: $\mathrm{Cl}=$ confidence interval, $\mathrm{OR}=$ odds ratio, ref = reference group 


\section{Interpretation}

In this study of more than 30000 patients with incident endstage renal disease in Canada, we found significant variation in the use of peritoneal dialysis in relation to the treating facility and the geographic region. The high degree of variation persisted after we accounted for a large number of patient- and facility-related factors that are known to influence use of peritoneal dialysis. Patients initiating dialysis in regions with high use of peritoneal dialysis, such as large urban centres, were significantly more likely to be started on peritoneal dialysis than patients in the lowest-use regions. In a publicly funded, universal health care system, this high level of measured variability in use of peritoneal dialysis at the facility and geographic level represents a possible area for improvement and may be amenable to health policy initiatives.

The large degree of unadjusted variability observed in this study could be explained by the presence of sicker patients at particular facilities or regions (i.e., case mix); however, significant variation persisted after we accounted for a large number of patient-related and facility-level factors. This result suggests that local and regional influences, such as physician preference, quality of predialysis care, availability of hemodialysis facilities and education about dialysis modalities may contribute to uptake of peritoneal dialysis. ${ }^{19,34}$ An important contributor to physician preference is the lack of evidence-based, standardized, "absolute" criteria for eligibility for peritoneal dialysis. In the absence of such evidence, opinion-based recommendations, local champions and advocates, and anecdote often serve as guides to eligibility, which may in turn contribute to the observed regional heterogeneity. This phenomenon was recently reported for 630 incident dialysis patients from a single provincial renal program. ${ }^{35}$ In that study, the adjusted odds ratio for starting patients on peritoneal dialysis ranged from 0.32 to 3.95 among peer nephrologists. This high degree of variability was surprising, considering that the physicians belonged to a shared provincial program with many standardized processes (excluding referral and eligibility for peritoneal dialysis). The variability among peer nephrologists ${ }^{35}$ was consistent with the results of a survey of nephrologists regarding patient suitability for peritoneal dialysis. ${ }^{36}$

Another important contributor to variation in use of different dialysis modalities is patient choice. Canada still allows patients who are medically suitable for any modality to select the dialysis modality. Other countries, including Hong Kong, have limited patient choice by implementing a "peritoneal dialysis first" strategy, whereby the government covers the costs of dialysis therapy only if patients choose peritoneal dialysis. ${ }^{37}$ This strategy has been successful, resulting in a prevalence of peritoneal dialysis of over $80 \%$ in Hong Kong, whereas in jurisdictions without a similar policy and the application of patient preferences, such as Canada and Europe, the historical prevalence of peritoneal dialysis is roughly $20 \%$ to $25 \%$. $^{37}$

We used the intraclass correlation coefficient to measure variability at the facility and geographic level, which allowed quantification of peritoneal dialysis use and comparisons between health care systems. In our study, the measured variability in peritoneal dialysis uptake at the facility level was comparable to other reported facility-level variations in

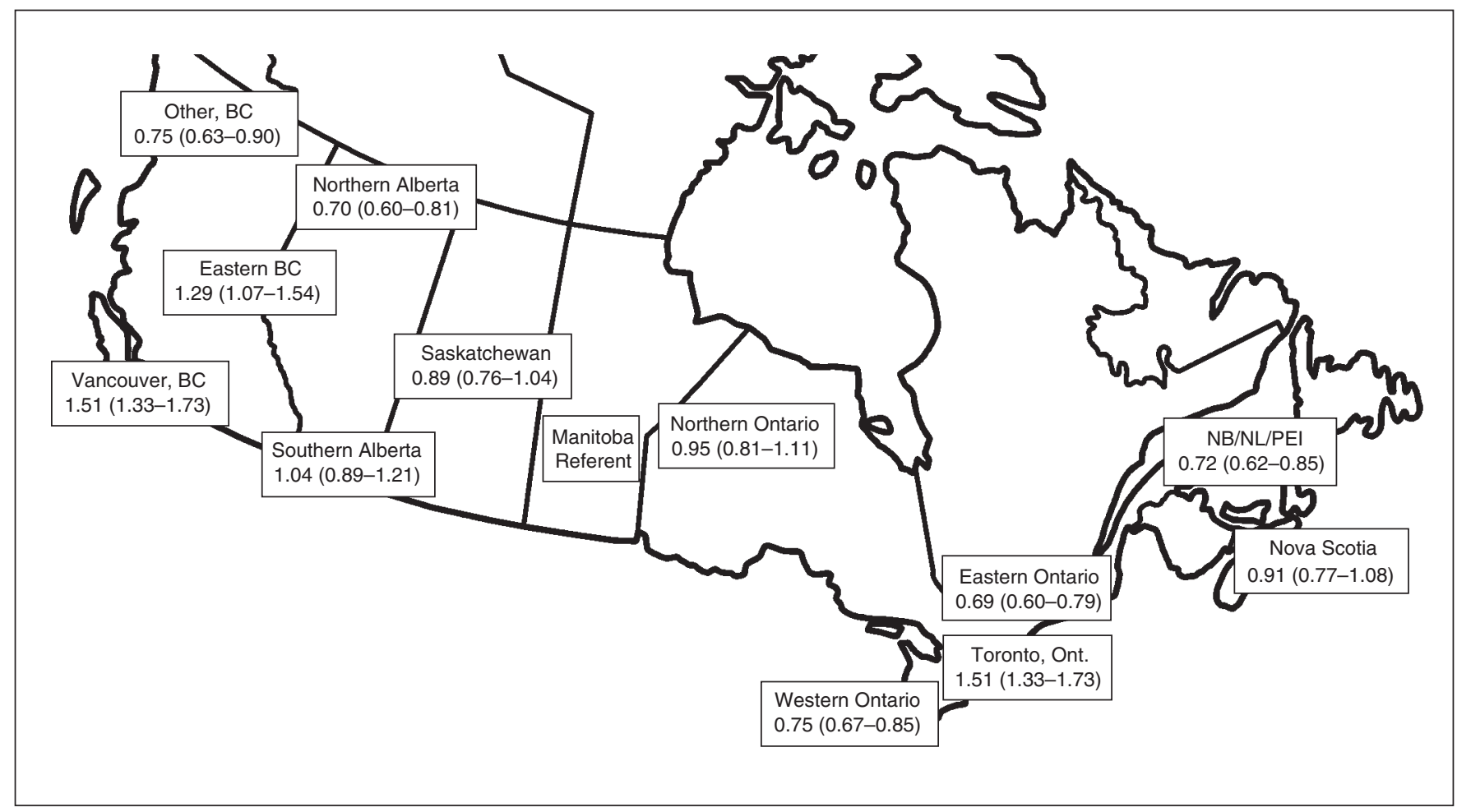

Figure 2: Adjusted odds ratios (with $95 \%$ confidence intervals) for use of peritoneal dialysis at 90 days across geographic regions of Canada. 
dialysis care. For example, 2 US studies reported facilitylevel variations of $7.6 \%$ in use of arteriovenous fistula ${ }^{38}$ and $11.5 \%$ in achievement of dialysis adequacy ${ }^{39}$ among 173 facilities. Numerous studies have achieved improvements in patient care metrics by targeting a decrease in variability through quality improvement programs..$^{40,41}$ Appropriate health care policies, such as national standardization of eligibility criteria for peritoneal dialysis and adoption of best practices for programs with high use of peritoneal dialysis, could be easily implemented and could be followed using national administrative data sets measuring changes in variability. Given that many countries collect national-level data for patients with end-stage renal disease, measures of regional variability in use of peritoneal dialysis could allow international comparisons and analysis of differences in patient comorbidities and demographic characteristics.

We observed a small but steady decline in use of peritoneal dialysis over the decade from 2001 to 2010. Similar (but larger) declines have been reported in large dialysis registries and globally. ${ }^{37,42}$ The decline that we observed may have been lower than that in other countries, possibly because of promotion of home modalities through physician remuneration, limitations on the availability of in-centre hemodialysis and strong education about peritoneal dialysis during residency and fellowship training. Nevertheless, the overall decline in peritoneal dialysis in Canada likely reflects, in part, the shift in demographic characteristics among patients with end-stage renal disease (older patients with an increasing number and severity of comorbid conditions). ${ }^{\prime}$

Numerous factors were associated with use of peritoneal dialysis, including younger age, fewer comorbid illnesses, predialysis care and greater distance from residence to dialysis facility. In the absence of assisted peritoneal dialysis programs, the physical and mental ability to perform the peritoneal dialysis procedure is a key rate-limiting step to its widespread use. Advancing age coupled with a high burden of comorbid illnesses would therefore limit its use. ${ }^{15,43}$ Use of peritoneal dialysis was significantly increased in association with relevant education and awareness, which in turn rely on access to nephrology care and services. Several studies have demonstrated that predialysis programs for patients with chronic kidney disease that incorporate education about home dialysis modalities are associated with greater uptake of peritoneal dialysis. ${ }^{15,44-47}$ Canada is a vast, geographically diverse country with many sparsely populated rural areas; however, the majority of hemodialysis facilities are located in larger, urban centres. It follows that residence in an area remote from such facilities would be associated with increased use of peritoneal dialysis. Previous studies have reported that lower numbers of peritoneal dialysis patients treated at a facility were associated with increases in technique failure and death. ${ }^{48}$ In our study, facility size was not significantly associated with use of peritoneal dialysis. An interesting observation was the ethnic disparities in peritoneal dialysis uptake, with use of peritoneal dialysis being low among Aboriginal patients and high among those of East Asian background. The reasons for these disparities are unclear, but they have been consistently described and may partially explain the higher use of peritoneal dialysis in the large, urban centres of Vancouver and Toronto. ${ }^{12,49}$

One potential barrier to the initiation of peritoneal dialysis is the requirement for insertion of a catheter, and access to resources for doing so may vary from region to region. This situation may partly explain the decline in variability of peritoneal dialysis use among dialysis centres with increased duration of dialysis. For example, variability among facilities was almost $2 \%$ lower for patients receiving peritoneal dialysis by 90 days after initiation of dialysis than for patients whose therapy began with peritoneal dialysis. Bedside insertion of peritoneal dialysis catheters by a nephrologist has improved use of peritoneal dialysis by circumventing possible surgical barriers. $^{50-52}$ Other issues leading to regional variation include availability of home care-assisted peritoneal dialysis programs, proportion of cases requiring emergent initiation of dialysis, physician reimbursement and in-centre capacity for intermittent hemodialysis. ${ }^{43,53,54}$

Our findings are strengthened by the use of multilevel (hierarchic) models that appropriately accounted for withinfacility and geographic region clustering, in contrast to the traditional logistic and linear regression models used in previous studies, which have consistently led to inflated effect estimates. ${ }^{31}$ We have reported the intraclass correlation coefficient, which is an increasingly recognized metric for describing variability in complex environments, such as health care systems. ${ }^{41,55}$ Our models accounted for a large number of patient- and facility-level characteristics, and our findings were consistent over a number of sensitivity analyses. We examined variation at the level of facilities rather than individual physicians, because many nephrology practices in Canada use shared-care models. Because Canada has universal, government-funded health care, this study of peritoneal dialysis use was not confounded by the presence of private (for-profit) dialysis services.

\section{Limitations}

Our study had certain limitations. We examined registry data and therefore lacked information about why patients who were not receiving peritoneal dialysis were deemed ineligible for this form of therapy (e.g., poor functional status, patient preference, cognitive capacity, socioeconomic factors). We also did not have information on individual physicians to determine whether observed variability was due to decisionmaking by individual physicians or systematic processes at the facility level. Information on satellite hemodialysis units was not captured, and we did not include patients from Quebec. We were unable to account for assisted peritoneal dialysis programs, which may make it easier for patients to use peritoneal dialysis, especially in urban areas.

\section{Conclusion}

Across Canada, the use of peritoneal dialysis varies considerably according to the treating facility and the geographic location. Because peritoneal dialysis offers comparable medical outcomes, improved quality of life and increased patient satisfaction, attempts to minimize regional and facility-level 
variation in the use of this form of dialysis seem reasonable. Although an optimal target remains unknown, increases in use of peritoneal dialysis achieved through health policy interventions could lead to substantial yearly cost savings to the health care system without diminishing and possibly improving patient outcomes. Coordinated plans to standardize and optimize implementation of peritoneal dialysis should be considered.

\section{References}

1. Canadian Organ Replacement Register annual report: treatment of end-stage organ failure in Canada, 2001 to 2010. Ottawa (ON): Canadian Institute for Health Information; 2011.

2. Ganesh SK, Hulbert-Shearon T, Port FK, et al. Mortality differences by dialysis modality among incident ESRD patients with and without coronary artery disease. 7 Am Soc Nephrol 2003;14:415-24.

3. Jaar BG, Coresh J, Plantinga LC, et al. Comparing the risk for death with peritoneal dialysis and hemodialysis in a national cohort of patients with chronic kidney disease. Ann Intern Med 2005;143:174-83.

4. McDonald SP, Marshall MR, Johnson DW, et al. Relationship between dialysis modality and mortality. 7 Am Soc Nephrol 2009;20:155-63.

5. Murphy SW, Foley RN, Barrett BJ, et al. Comparative mortality of hemodialysis and peritoneal dialysis in Canada. Kidney Int 2000;57:1720-6.

6. Vonesh EF, Moran J. Mortality in end-stage renal disease: a reassessment of differences between patients treated with hemodialysis and peritoneal dialysis. 7 Am Soc Nephrol 1999; 10:354-65.

7. Winkelmayer WC, Glynn RJ, Mittleman MA, et al. Comparing mortality of elderly patients on hemodialysis versus peritoneal dialysis: a propensity score approach. $7 \mathrm{Am}$ Soc Nephrol 2002;13:2353-62.

8. Wu AW, Fink NE, Marsh-Manzi JVR, et al. Changes in quality of life during hemodialysis and peritoneal dialysis treatment: generic and disease specific measures. 7 Am Soc Nepbrol 2004;15:743-53.

9. Rubin HR, Fink NE, Plantinga L, et al. Patient ratings of dialysis care with peritoneal dialysis vs hemodialysis. 7AMA 2004;291:697-703.

10. Liao CT, Shiao CC, Huang JW, et al. Predictors of faster decline of residual renal function in Taiwanese peritoneal dialysis patients. Perit Dial Int 2008;28 Suppl 3:S191-5.

11. Sood MM, Hemmelgarn B, Rigatto C, et al. Association of modality with mortality among Canadian Aboriginals. Clin 7 Am Soc Nephrol 2012;7:1988-95.

12. Hemmelgarn BR, Chou S, Wiebe N, et al. Differences in use of peritoneal dialysis and survival among East Asian, Indo Asian, and white ESRD patients in Canada. Am $\mathcal{F}$ Kidney Dis 2006;48:964-71.

13. Woodrow G. What are the factors underlying the variation in the use of peritoneal dialysis? Nephrol Dial Transplant 2013;28:501-4.

14. Stack AG, Molony DA, Rahman NS, et al. Impact of dialysis modality on survival of new ESRD patients with congestive heart failure in the United States. Kidney Int 2003;64:1071-9.

15. Jager KJ, Korevaar JC, Dekker FW, et al. The effect of contraindications and patient preference on dialysis modality selection in ESRD patients in the Netherlands. Am 7 Kidney Dis 2004;43:891-9.

16. Tonelli M, Hemmelgarn B, Culleton B, et al. Mortality of Canadians treated by peritoneal dialysis in remote locations. Kidney Int 2007;72:1023-8.

17. Allen N, Schwartz D, Sood AR, et al. Perceived barriers to guidelines in peritoneal dialysis. Nephrol Dial Transplant 2011;26:1683-9.

18. McClellan WM, Wasse H, McClellan AC, et al. Treatment center and geographic variability in pre-ESRD care associate with increased mortality. $7 \mathrm{Am}$ Soc Nephrol 2009;20:1078-85.

19. Kutner NG, Zhang R, Huang Y, et al. Patient awareness and initiation of peritoneal dialysis. Arch Intern Med 2011;171:119-24.

20. Guo A, Mujais S. Patient and technique survival on peritoneal dialysis in the United States: evaluation in large incident cohorts. Kidney Int Suppl 2003; (88):S3-12.

21. Mendelssohn DC, Langlois N, Blake PG. Peritoneal dialysis in Ontario: a natural experiment in physician reimbursement methodology. Perit Dial Int 2004; 24:531-7.

22. Moist LM, Richards HA, Miskulin D, et al. A validation study of the Canadian Organ Replacement Register. Clin 7 Am Soc Nephrol 2011;6:813-8.

23. Vincenty T. Direct and inverse solutions of geodesics on the ellipsoid with application of nested equations. Surv Rev 1975;176:88-93.

24. Tonelli M, Manns B, Culleton B, et al. Association between proximity to the attending nephrologist and mortality among patients receiving hemodialysis. CMA7 2007;177:1039-44.

25. Mendelssohn DC, Pisoni RL, Arrington CJ, et al. A practice-related risk score (PRS): a DOPPS-derived aggregate quality index for haemodialysis facilities. Nephrol Dial Transplant 2008;23:3227-33.

26. Benner D, Hollister D, McAllister CJ, et al. The DaVita Quality Index (DQI): a measure of clinical performance. Dial Transplant 2003;32:269-73.
27. Schaubel DE, Morrison HI, Desmeules M, et al. End-stage renal disease in Canada: prevalence projections to 2005. CMA7 1999;160:1557-63.

28. Rocco MV, Frankenfield DL, Hopson SD, et al. Relationship between clinical performance measures and outcomes among patients receiving long-term hemodialysis. Ann Intern Med 2006;145:512-9.

29. Rodriguez G, Elo I. Intra-class correlation in random-effects models for binary data. Stata 7 2003;3:32-46.

30. Heo M, Leon A.C. Performance of a mixed effects logistic regression model for binary outcomes with unequal cluster size. F Biopharm Stat 2005;15:513-26.

31. Greenland S. Principles of multilevel modelling. Int F Epidemiol 2000;29:158-67.

32. Snijders TBR, Bosker RJ. Multilevel analysis: an introduction to basic and advanced multilevel modeling. London (UK): Sage Publications Ltd.; 1990.

33. Schafer JL. Multiple imputation: a primer. Stat Methods Med Res 1999;8:3-15.

34. Blake P. Proliferation of hemodialysis units and declining peritoneal dialysis use: an international trend. Am 7 Kidney Dis 2009;54:194-6.

35. Hingwala J, Diamond J, Tangri N, et al. Underutilization of peritoneal dialysis: the role of the nephrologist's referral pattern. Nephrol Dial Transplant 2013; 28:732-40.

36. Little J, Irwin A, Marshall T, et al. Predicting a patient's choice of dialysis modality: experience in a United Kingdom renal department. Am 7 Kidney Dis 2001;37:981-6.

37. Jain AK, Blake P, Cordy $\mathrm{P}$, et al. Global trends in rates of peritoneal dialysis. $7 \mathrm{Am}$ Soc Nephrol 2012;23:533-44.

38. Tangri N, Moorthi R, Tighiouhart H, et al. Variation in fistula use across dialysis facilities: Is it explained by case-mix? Clin 7 Am Soc Nephrol 2010;5:307-13.

39. Tangri N, Tighiouart H, Meyer KB, et al. Both patient and facility contribute to achieving the Centers for Medicare and Medicaid Services' pay-for-performance target for dialysis adequacy. 7 Am Soc Nephrol 2011;22:2296-302.

40. Fink JC, Zhan M, Blahut SA, et al. Measuring the efficacy of a quality improvement program in dialysis adequacy with changes in center effects. $7 \mathrm{Am} \mathrm{Soc}$ Nephrol 2002;13:2338-44.

41. Howley PP, Gibberd R. Using hierarchical models to analyse clinical indicators: a comparison of the gamma-Poisson and beta-binomial models. Int 7 Qual Health Care 2003;15:319-29.

42. United States Renal Data System. 2009 annual data report. Vol. 2. Atlas of end-stage renal disease in the United States. Bethesda (MD): National Institutes of Health, National Institute of Diabetes and Digestive and Kidney Diseases; 2009.

43. Oliver MJ, Garg AX, Blake PG, et al. Impact of contraindications, barriers to self-care and support on incident peritoneal dialysis utilization. Nephrol Dial Transplant 2010;25:2737-44.

44. Marrón B, Martinez Ocana J, Salgueira M, et al. Analysis of patient flow into dialysis: role of education in choice of dialysis modality. Perit Dial Int 2005;25 Suppl 3:S56-S9.

45. Klang B, Björvell H, Clyne N. Predialysis education helps patients choose dialysis modality and increases disease-specific knowledge. F Adv Nurs 1999;29:869-76.

46. Goovaerts T, Jadoul M, Goffin E. Influence of a pre-dialysis education programme (PDEP) on the mode of renal replacement therapy. Nephrol Dial Transplant 2005;20:1842-7.

47. Manns BJ, Taub K, VanderStraeten C, et al. The impact of education on chronic kidney disease patients' plans to initiate dialysis with self-care dialysis: a randomized trial. Kidney Int 2005;68:1777-83.

48. Schaubel DE, Blake PG, Fenton SSA. Effect of renal center characteristics on mortality and technique failure on peritoneal dialysis. Kidney Int 2001;60:1517-24.

49. Tonelli M, Hemmelgarn B, Manns B, et al. Use and outcomes of peritoneal dialysis among Aboriginal people in Canada. 7 Am Soc Nephrol 2005;16:482-8.

50. Zaman F. Peritoneal dialysis catheter placement by nephrologist. Perit Dial Int 2008;28:138-41.

51. Goh BL, Ganeshadeva YM, Eng Chew S, et al. Does peritoneal dialysis catheter insertion by interventional nephrologists enhance peritoneal dialysis penetration? Semin Dial 2008;21:561-6.

52. Asif A, Byers P, Gadalean F, et al. Peritoneal dialysis underutilization: the impact of an interventional nephrology peritoneal dialysis access program. Semin Dial 2003;16:266-71.

53. Troidle L, Kliger A, Finkelstein F. Barriers to utilization of chronic peritoneal dialysis in network \#1, New England. Perit Dial Int 2006;26:452-7.

54. Just PM, de Charro FT, Tschosik EA, et al. Reimbursement and economic factors influencing dialysis modality choice around the world. Nephrol Dial Transplant 2008;23:2365-73

55. Fung V, Schmittdiel JA, Fireman B, et al. Meaningful variation in performance: a systematic literature review. Med Care 2010;48:140-8.

Affiliations: Ottawa Hospital Research Institute and the University of Ottawa (Sood), Ottawa, Ont.; Seven Oaks Hospital and University of Manitoba (Tangri, Rigatto, Komenda), Winnipeg; Cardiac Sciences Program (Hiebert), St. Boniface Hospital, Winnipeg, Man.; Saskatoon Health Region and University of Saskatchewan (Kappel), Saskatoon, Sask.; Health Sciences Centre and University of Manitoba (Dart), Winnipeg, Man.; St. Paul's Hospital and the University of British Columbia (Levin), Vancouver, BC; Foothills Hospital and University of Calgary (Manns), Calgary, Alta.; the Faculty of Nursing (Molzahn), University of Alberta, 
Edmonton, Alta.; Sunnybrook Health Sciences Centre and University of Toronto (Naimark), Toronto, Ont.; Jewish General Hospital and McGill University (Nessim), Montréal, Que.; Dalhousie University (Soroka), Halifax, NS; McGill University Health Centre and McGill University (Zeppitelli), Montréal, Que.

Contributors: All authors contributed to study conception and design. Manish Sood drafted the first version of the manuscript and is responsible for the data integrity and analysis. Brett Hiebert performed the statistical analysis. Paul Komenda, Navdeep Tangri, David Naimark, Sharon Nessim, Claudio Rigatto, Adeera Levin, Braden Manns, Anita Molzahn, Joanne Kappel, Brett Hiebert, Michael Zappitelli, Allison Dart and Steven
Soroka critically revised and added to the manuscript. All of the authors reviewed the article and approved the final version for publication.

Funding: This study was supported by the Canadian Institutes of Health Research and the Kidney Foundation of Canada. The study sponsors had no role in the design of the study, the collection, analysis or interpretation of data, the writing of the report or the decision to submit the article for publication.

Supplemental information: For reviewer comments and the original submission of this manuscript, please see www.cmajopen.ca/content/2/1 /E36/suppl/DC1 